\title{
Newly-Discovered Anomalies in Galactic Cosmic Rays: Time for Exotic Scenarios?
}

\begin{abstract}
Recent observations of galactic cosmic rays (CR) in the 1-500 GeV energy range have revealed striking deviations from what deemed "standard." The anomalies cut across hadronic and leptonic CRs. I discuss findings that challenge physical mechanisms long held responsible for the CR production in galactic supernova remnants (SNR). I also consider some new physics of particle acceleration in SNR shocks that is not part of conventional models but may explain the anomalies. However, a possible 20-30\% excess remains unaccounted for in the $e^{+} / e^{+}$ratio over the range of a few $100 \mathrm{GeV}$. If not explained by future models, it suggests an additional source of positrons such as a dark matter decay/annihilation or pulsar contribution. Earlier efforts to explain both the $e^{+} / e^{-}$and $p / \mathrm{He}$ anomalies with the "standard" models by adjusting the SNR environmental parameters and multiple sources are critically assessed.
\end{abstract}

Keywords: cosmic rays, supernova remnants, particle acceleration, dark matter PACS: 98.38.Mz, 98.70.Sa, 96.50.S-, 95.35.+d

\section{Recent Challenges}

In the two years passed after the first "Cosmic Ray Origin-Beyond the Standard Model" San Vito Conference [1], new discoveries moved the CR modeling farther beyond all "standards" than perhaps over the preceding decade, which was also rich in surprises. Some models explaining the data were jettisoned after the next data release or a careful analysis of those available [2]. The latest debates seem to refocus on a perplexing rise of the $e^{+} / e^{-}$fraction in the $10-300 \mathrm{GeV}$ range, and possibly related flatness of $\bar{p} / p$ and $e^{+} / p$ ratios. They gained widespread interest after the AMS-02 team has published the most accurate elemental and energy spectra in this range [3].

Anomalies in the CR spectra and composition are becoming a general trend in $\mathrm{CR}$ observations. Besides the $e^{+} / e^{-}$anomaly, there is at least one interesting phenomenon that merits a short discussion, as it may also help to understand the $e^{+}$excess.

\subsection{Proton to Helium, Carbon, and Oxygen ratios}

In 2011 Pamela experiment [4] revealed a remarkable 0.1 index difference between the proton and $\mathrm{He}$ rigidity spectra in 2-200 GV range. The limits here are contingent rather than intrinsic to the original spectrum, merely set by the solar modulation and instrument's range. The scaling is likely to continue beyond this range, according to high-energy observations [5].

With this finding, the Pamela team caught the Holy Grail of the CR origin hypothesis, the diffusive shock acceleration (DSA) mechanism in the cross-hair. It is widely believed to supply the bulk of galactic CR from their presumed production sites in supernova remnant (SNR) shocks. To appreciate the threat to the DSA, consider equations of motion for a particle in electromagnetic fields $\mathbf{E}(\mathbf{r}, t)$ and $\mathbf{B}(\mathbf{r}, t)$, using the particle rigidity $\mathcal{R}=\mathbf{p} c / e Z$ instead of its momentum $\mathbf{p}(Z$ is the charge number), e.g., [6]:

$$
\frac{1}{c} \frac{d \boldsymbol{R}}{d t}=\mathbf{E}(\mathbf{r}, t)+\frac{\mathcal{R} \times \mathbf{B}(\mathbf{r}, t)}{\sqrt{\mathcal{R}_{0}^{2}+\mathcal{R}^{2}}},
$$




$$
\frac{1}{c} \frac{d \mathbf{r}}{d t}=\frac{\mathcal{R}}{\sqrt{\mathcal{R}_{0}^{2}+\mathcal{R}^{2}}} .
$$

The fields $\mathbf{E}(\mathbf{r}, t)$ and $\mathbf{B}(\mathbf{r}, t)$ are arbitrary, so our inference below will apply to both the acceleration and propagation of the CRs. It follows that all species with $\mathcal{R} \gg \mathcal{R}_{0}=A m_{p} c^{2} / Z e$ ( $A$ is the atomic number and $m_{p^{-}}$ proton mass, so $\left.\mathcal{R}_{0} \sim A / Z \mathrm{GV}\right)$, have identical orbits in the phase space $(\mathbf{r}, \mathcal{R})$.

Let us select, at the moment $t=0$, a group of $\mathrm{H}^{+}$and $\mathrm{He}^{++}$ions undergoing shock acceleration from the same $\mathcal{R}(0) \gg \mathcal{R}_{0}$. Let their ratio at $t=0$ be $\eta=N_{p} / N_{\mathrm{He}}$. According to eqs. (1) and (2), it is conserved during the $a c$ celeration and propagation. Physically, $\eta$ may depend on $\mathcal{R}(0)$, only if the strong inequality $\mathcal{R}(0) \gg \mathcal{R}_{0}$ is violated. But, as long as $\eta(t=0)$ is fixed and the acceleration is stationary, we can assign $\eta$ to a different $\mathcal{R}=\mathcal{R}_{1} \gg \mathcal{R}_{0}$ beyond which the above argument about conservation of $\eta$ applies.

The individual values of $N_{p}(\mathcal{R})$ and $N_{\mathrm{He}}(\mathcal{R})$ will decline with growing $\mathcal{R}$ since particles gradually escape the accelerator and then galaxy. But the ratio $N_{p} / N_{\mathrm{He}}$ must remain constant because the escape mechanisms are the same for $p$ and He. As we know, $N_{p} / N_{\mathrm{He}}$ does depend on $\mathcal{R}$, nonetheless. Two effects can be responsible for that: spallation and time-variability of injection. We will use the rigidity dependence of the fractions of different species as a primary probe into the intrinsic properties of CR accelerators. Unlike the individual spectra, the fractions are unaffected by the CR propagation, reacceleration, and losses from the galaxy.

Meanwhile, the spallation effects can hardly account for the $p / \mathrm{He}$ rigidity dependence [2]. Furthermore, the rigidity independence of acceleration and propagation rules out "easy solutions." The $0.1 \mathrm{p} / \mathrm{He}$ index cannot be caused by propagation effects, second-order Fermi (re)acceleration, or compression-expansion cyclic acceleration in the ISM (interstellar medium) turbulence, contrary to several statements made in the literature along these lines. Accepting the obvious, let us leave alone scenarios that are inconsistent with the dynamical equivalence of $p$ and $\mathrm{He}$ ions at $\mathcal{R} \gg \mathcal{R}_{0}$.

More recently, also the $p / C$ and $p / O$ ratios have been measured to have the same rigidity scaling as $p / \mathrm{He}$, that is $\propto \mathcal{R}^{-0.1} 1$ Constant $\mathrm{C} / \mathrm{O}$ and $\mathrm{He} / \mathrm{C}$ ratios are expected from eqs.(1) and (2), provided that these elements are injected in a fully ionized state (see $\mathrm{Sec} 3.2$ though). Therefore, $\mathrm{He}, \mathrm{C}$, and $\mathrm{O}$ are likely to share their acceleration and propagation history, so it is unlikely that $\mathrm{C}$

\footnotetext{
${ }^{1}$ http://www.ams02.org/wp-content/uploads/2016/12/Final.pdf
}

and $\mathrm{O}$ are pre-accelerated from grains [7]. Moreover, the equivalence between the $\mathrm{He}, \mathrm{C}$ and $\mathrm{O}$ spectra corroborates an earlier conclusion [2] that spallation effects are insufficient to account for the observed differences in rigidity spectra between $p$ and other elements with similar $A / Z$.

\subsection{Positron anomaly}

The condition $\mathcal{R} \gg \mathcal{R}_{0}$ for the equivalence between $p$ and He is irrelevant for $e^{+}$and $e^{-}$, particles of equal mass, whereas the charge sign now matters most. If $e^{+}$and $e^{-}$are injected into the same or similar shocks, their spectral differences (by contrast to the secondary positrons ${ }^{2}$ must come from charge-sign sensitive acceleration and/or propagation. Most mechanisms for injection, acceleration, and propagation rely on particle interactions with MHD waves, self-driven by the particles or preexisting. However, such interactions do not seem to offer any distinct charge-sign dependence.

Much can be learned about wave-particle interactions from a linear resonance condition $\omega-k_{\|} v_{\|}=n \omega_{c}$. Here $\omega$ and $k_{\|}$are the frequency and wave-vector projection on the averaged magnetic field direction, $v_{\|}$is that of the particle velocity. The particle charge sign enters $\omega_{c}=$ $e B_{0} / \gamma m c$, but the integer $n$ can be positive/negative (for normal/anomalous Doppler resonance), or zero (for Cerenkov resonance). In the latter case the interaction is charge-sign independent, so we focus on the cyclotron resonances, $n= \pm 1$. Consider an individual, circularly polarized wave propagating along the field line. In its own frame, where the particle speed is $v_{\|}^{\prime}=v_{\|}-\omega / k_{\|}$, $\mathbf{E}=0$ and the particle perceives only the Lorenz force. The resonance occurs when this force oscillates in sync with the particle gyromotion, $k_{\|} v_{\|}^{\prime}= \pm \omega_{c}$. The choice of the sign $( \pm)$ depends on the wave polarization while, physically, the condition means that the particle perceives a non-oscillating force (strong effect).

Consequently, once protons resonantly generate waves of a particular polarization, these waves will resonantly interact with positrons with the same $v_{\|}^{\prime} / \omega_{c}$ and, also with electrons moving at $-v_{\|}^{\prime}$. These considerations are not strictly correct if the wave grows to make the resonance condition nonlinear [8]. Such a situation occurs near a collisionless shock transition to which we return later. Here we note that under typical DSA conditions when $v_{\|} \gg U_{\text {shock}}$, particles are isotropic in pitch angle. The isotropy implies a nearly equal amount of them with

\footnotetext{
${ }^{2}$ Note that the injected positrons can be seeded as secondaries, which we assume to be the case and discuss in $\operatorname{Sec} 4$
} 

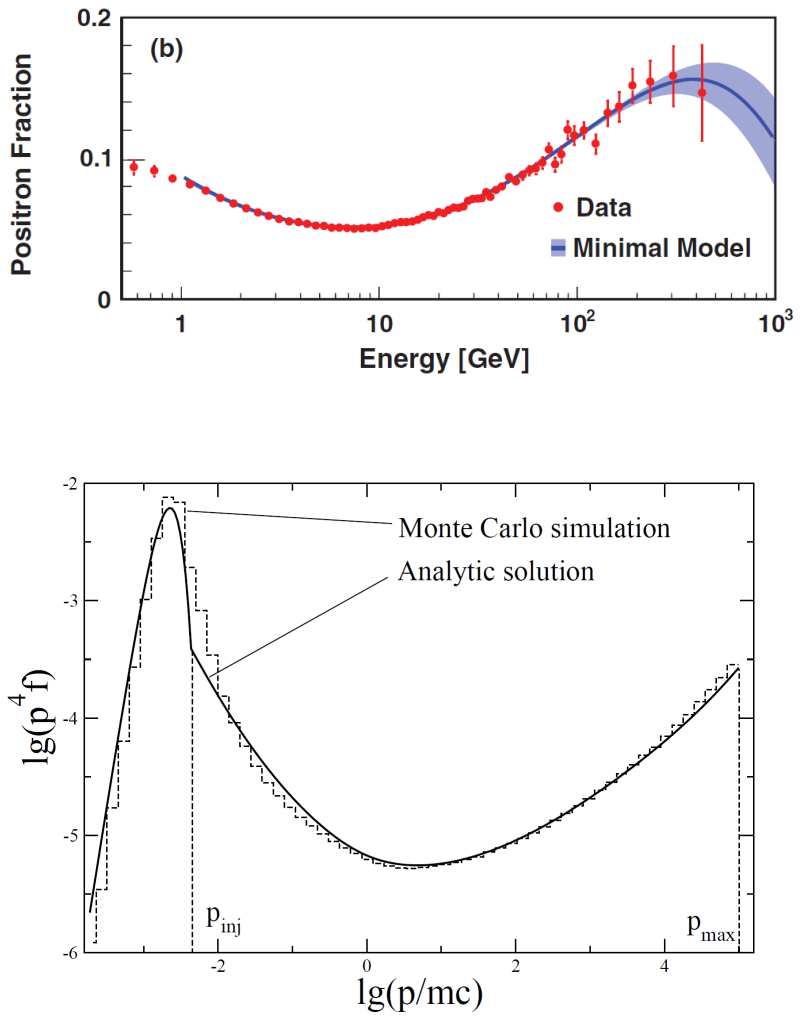

Figure 1: Top Panel: $e^{+} /\left(e^{+}+e^{-}\right)$fraction measured by AMS-02 [9]. Bottom Panel: Analytic solution obtained in [10] for NLDSA of protons and adopted from [11] (shown together with Monte Simulations from [12]).

positive and negative $v_{\|}^{\prime}$, so it is hard to expect a significant suppression of electron acceleration compared to the positrons.

However plausible this conclusion may seem, the $e^{+} /\left(e^{+}+e^{-}\right)$ratio strongly depends on energy [13, 14] (Fig 1, top panel). Moreover, by contrast with the $\mathrm{He} / p$ ratio that shows a featureless scaling $\propto \mathcal{R}^{0.1}$ (at least in the $\mathcal{R}>\mathcal{R}_{0}$ range) the $e^{+} /\left(e^{+}+e^{-}\right)$ratio possesses one or possibly even two extrema. Strong growth at high energies attracted the most attention, but a distinct minimum at $\approx 8 \mathrm{GeV}$ and descending branch in the range $E<8 \mathrm{GeV}$ may equally be relevant. This nonmonotonic positron fraction is hard to explain with a minimum of assumptions. It appears particularly at odds with a single source DSA operation in an SNR, which predicts similar rigidity spectra for all species accelerated in the source. The data indicate that this ratio has a trend towards saturation at $200-400 \mathrm{GeV}$, or may even decline beyond this energy range. So, a maximum in this range is also possible which, of course, has not passed unnoticed by the dark matter hunters.

\section{Possible Physics behind the anomalies}

Both $e^{+}$and He spectral anomalies violate the equal rigidity rule, whereby two particles are dynamically indistinguishable if they have equal rigidities, regardless of mass or charge. In the first example, the difference between $p$ and $\mathrm{He}$ was in their rest-mass rigidity, namely $\mathcal{R}_{0, \mathrm{He}}=2 \mathcal{R}_{0, p}\left(\mathcal{R}_{0, \mathrm{He}}=\mathcal{R}_{0, \mathrm{C}}=\mathcal{R}_{0, \mathrm{O}}\right.$, for fully ionized ions). It explicitly enters the equations of motion but becomes unimportant when particles are accelerated to $\mathcal{R} \gg \mathcal{R}_{0}$. From this moment on their rigidity spectra (apart from normalization) must be the same, which they are not. In the second example, the absolute value of rigidity was the same but the signs were opposite $\left(e^{ \pm}\right)$. Accepting the arguments for charge-sign symmetry of wave-particle interaction, the most likely mechanism behind the $e^{ \pm}$separation during or before the acceleration phase is a macroscopic electric field. Indeed, an accelerator-scale electric field can turn positrons toward it while fending off the electrons, or vice versa. A legitimate concern about a plasma quasineutrality in such field is not a dogma but rather a problem in plasma physics.

The positron excess also hints at an exciting but still speculative involvement of weakly interacting massive particles (WIMPs). The WIMPS are widely believed to be present in the dark sector of the particle theories beyond the standard model [15, 16]. The search for them in CR data is argued to be in a synergistic relation with that in the LHC. Decay or annihilation of such particles has been suggested as a possible explanation for the rise in the $e^{+} / e^{-}$ratio, as well as surprisingly flat (for the secondaries) $\bar{p} / p$ ratio, Fig 2 .

Let us lay aside the WIMP scenarios for a while. Earlier considerations give enough clues as to where should one look for the explanations. In the case of the $\mathrm{p} / \mathrm{He}$ anomaly, the particle segregation must occur during the non-relativistic phase of acceleration. Ideally, it must be part of the shock injection mechanism at $\mathcal{R} \ll \mathcal{R}_{0}$. This would be the way to produce relatively more He than protons with growing rigidity. However, this alone will not solve the problem, if the shock accelerates particles under stationary conditions, first of all at a constant Mach number. Fortunately, the Mach number of an SNR shock decreases in time, and the mechanism may work. Of course, it requires the relative injection efficiency of $\mathrm{p} / \mathrm{He}$ to be also Mach number dependent which is true.

In the case of the $e^{+} / e^{-}$anomaly, a macroscopic electric field can separate the species very efficiently. Be- 


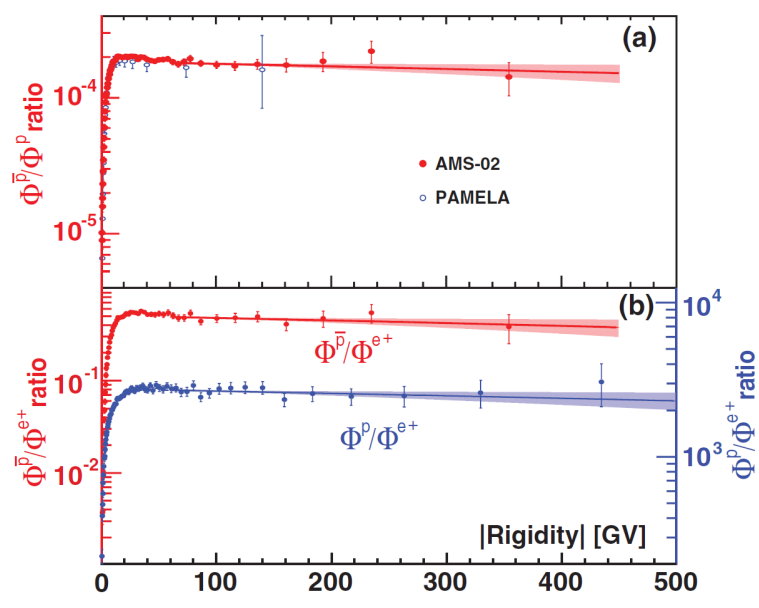

Figure 2: $\bar{p} / p$ and $e^{+} / p$ fractions measured by AMS-02 and Pamela.

low, we consider $p / \mathrm{He}$ and $e^{+} / e^{-}$spectral anomalies independently and compare the physical mechanisms outlined above with environmental mechanisms, suggested in the literature.

\section{Modeling of $p /$ He Spectrum}

The rigidity-dependent $p /$ He ratio seems paradoxical from the perspective of a single accelerator but makes more sense if the $p$ and He spectra come from two or more sources.

\subsection{Environmental and multisource scenarios}

Multisource models (see [17] for a recent review) were suggested before the high-quality measurements [4, 18] have become available. The models were adjusted for the newest data [19], but their intrinsic limitations in accounting for the persistent 0.1 difference between the $\mathrm{He}$ and $p$ indices remain and can be readily assessed. For instance, if only two sources with fixed power-law indices and normalization for $\mathrm{H}$ and $\mathrm{He}$ contribute to the observed spectrum, they cannot generate a nearly constant slope of $N_{p} / N_{\mathrm{He}} \propto \mathcal{R}^{-0.1}$ over two decades in rigidity (at least!). This slope can occur only in a transition region between two regions each of which corresponds to one particular source with a constant slope. By adding more sources and adjusting propagation parameters [19] one can extend the transition region somewhat. However, unless the sources with the hardest spectra are proton deficient, it is hard to imagine that $\mathrm{He}$ and heavier elements approach the observed proton flux in the knee region [20]. They must maintain a consistently harder spectrum relative to protons for all $\mathcal{R} \gg \mathcal{R}_{0}$ which is impossible if only one stationary source dominates the knee region.

A different scenario, which may operate at a single SNR exploding into a superbubble, assumes an inhomogeneity of an SNR shock environment with an outward increasing $\mathrm{H} / \mathrm{He}$ ratio [7]. The idea is that as the shock expands into such an environment, the ratio of the processed H/He ions also increases. Since the shock weakens with growing radius, the integrated $\mathrm{H}$ spectrum will be softer than that of He. However, to generate a 0.1 difference in the spectral indices, the radial dependence of the He abundance must be coordinated with the shock Mach number $M$ dependence on its radius, $M(R)$, since the power-law index $q=4 /\left(1-M^{-2}\right)$ is the same for all species. While not impossible for $\mathrm{He}$, the same radial profile of $\mathrm{C}$ and $\mathrm{O}$ abundance seems improbable. Recall, that all three elements have been measured to have identical spectra. Besides, the explosion must occur near the maximum of the $\mathrm{He}, \mathrm{C}$, and $\mathrm{O}$ abundances. Had it occurred in the region where the abundance changes monotonically, the effect would not be pronounced. The latter concern may be resolved by assuming a layered pre-supernova wind structure [21], but again, He, C, and $\mathrm{O}$ need to have proper radial profiles.

\subsection{Shock's intrinsic mechanism}

The above mechanisms are contingent in that they depend on how the CR sources are distributed in the ISM, types of SN progenitor stars, or their environments. But can a shock change an element abundance by selectively accelerating it out of a homogeneous background plasma? It has been argued for some time [8] that precisely this occurs in quasi-parallel shocks. What is relevant to the present discussion, the selectivity mechanism significantly depends on the shock Mach number.

At an intuitive level, the main argument is of a Le Chatelier's type. Injected protons distort the shock structure in such a way as to reduce the injection. Quasiparallel shocks thus promote the CR diversity by disfavoring acceleration of the most abundant species. The segregation mechanism works as follows. Injected protons drive unstable Alfven waves in front of the shock. These waves control the injection of all particles by regulating their access to those parts of the phase space from where they can cross and re-cross the shock. As protons drive these waves, the waves trap protons most efficiently. Furthermore, the waves are almost frozen into the local fluid so, when crossing the shock interface, they entrain most particles and prevent them from escaping upstream, thus significantly reducing their odds for injection. Again, most efficient is namely 


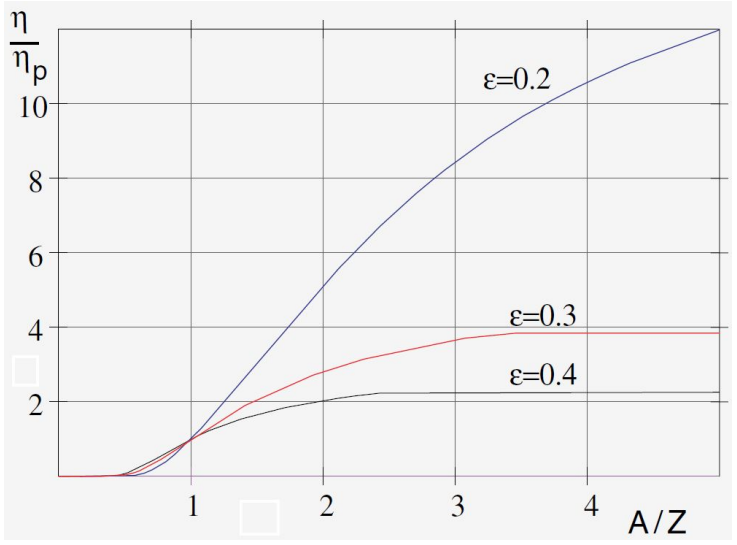

Figure 3: Elemental injection efficiencies normalized to proton efficiency depending on mass to charge ratio $\mathrm{A} / \mathrm{Z}$, shown for different amplitude parameters $\epsilon=B_{0} / \delta B_{2}$, where $\delta B_{2} \simeq r \delta B_{1}$ is the shockcompressed (compression ratio $r$ ) wave amplitude. For strong shocks $\epsilon \sim 1 / 3$ and it slowly decreases with the Mach number (adopted from [8]).

the proton entrainment, while, e.g., alpha particles have somewhat better chances to escape upstream and to get eventually injected. The wave trapping of alphas is weaker because of the mismatched wavelengths generated by the protons since their mass to charge ratios are different by a factor of two. The trapping becomes naturally stronger with growing wave amplitude, and this trend is more pronounced for the protons. This difference is the crux of the injection selectivity.

It follows that the injection rate must depend on the mass to charge ratio, $A / Z$. The charge-sign dependence is less significant, so we discuss it later. The injection efficiency is shown in Fig 3 as a function of $A / Z$ for a few values of the wave amplitude parameter $\epsilon=B_{0} / \delta B<1$ downstream. The latter quantity is thus determined by the amplitude of the wave generated by upstream protons undergoing injection (shock reflected or leaking from downstream). If the elements under consideration are fully ionized before injection, $\mathrm{He}, \mathrm{C}$, and $\mathrm{O}$ are injected at the same rate since they have similar $\mathcal{R}_{0}$. However, even if $\mathrm{C}$, for example, is not fully ionized, thus having a larger $A / Z$, it will not make much difference, as the injection curves saturate rather quickly. Therefore, an incomplete ionization will likely produce only an insignificant increase of the carbon injection. Moreover, when the shock Mach number is high, the assumption about a fully ionized plasma appears reasonable. Also, the validity range of the curves shown in Fig 3 is limited from above. If $A / Z$ increases significantly, such species becomes unaffected by the proton-driven waves and their injection will drop (not just saturated, as shown) because they will remain downstream after the first shock passing. Therefore, injection of grains with $A / Z \gg 1$ is unlikely. An ad hoc assumption about the presence of long waves generated by CRs at a knee rigidity $\left(\gtrsim 10^{14} \mathrm{GV}\right)$ is needed to thermalize such heavy grains and get them injected [22]. It is clear that waves generated upstream by injected protons are much more powerful than those long waves required to scatter grains, so we focus on the short waves.

The presence of such strong waves, significantly amplified upon shock crossing is well established by observations and simulations [23]. However, it is not clear what is the primary source of the wave driving protons. One physically motivated source of such particles is an evaporation of hot downstream plasma into the upstream medium, as suggested by Parker [24] (also called the thermal leakage). This scenario has been confirmed by early 1D hybrid simulations and observations, although the contribution of the ions backscattered from the shock interface has been recognized as well [23]. Several later simulations have favored backscattered (shock reflected, or shock dwelling) particles over those leaking from downstream [25, 26]. The distinction is somewhat semantic, as it is not easy to draw the line between these groups of particles. The backscattering occurs from a blurred and wiggling shock interface. For the thermal leakage to occur, the thermalization of downstream ions must be fast and close to completion. Therefore, the turbulence must be well developed to ensure strong wave-particle interaction with an extended resonance overlapping. These conditions are crucial for the entropy production, e.g. [27], not easy to meet in simulations.

There are many numerical limitations. To those given, e.g., in [25], we add a restricted number of wave modes and their harmonics available for the particle thermalization downstream. Wave spectra are artificially constrained by the size of the simulation box and its aspect ratio, thus impeding both the turbulence development and particle scattering. The list of limitations can be continued, but it is enough to say that a suppression of the nonlinear Landau damping, caused by the sparse wave spectrum, will slow down the thermalization. Recall that it requires a beating wave resonance with particles. A scarce number of particles in cell only contributes to this problem. We also note here that this type of wave-particle interaction should be particularly important for the entropy production downstream since the linear (cyclotron) resonance with Alfven waves results primarily in pitch-angle diffusion with almost no 
change in particle energy. If there is no full thermalization downstream, the thermal leakage may also be suppressed. However, the "numerical" shock may compensate for the resulting deficit of backstreaming ions by an enhanced reflection.

The particle reflection cannot be fully understood from the hybrid simulation as the electron kinetic effects are important [28, 29]. The PIC codes are still limited in the duration of acceleration, thus making the conclusions about the downstream thermalization mechanisms and the origin of backstreaming particles not compelling. For example, if all the injected protons were indeed shock reflected, as frequently suggested by simulations, it would not be easy to explain even a few reflected $\mathrm{H}^{++}$, given that only a limited fraction of incoming protons can be reflected in any event. Obviously, the both species could not be simultaneously reflected by a stationary reflecting barrier, be it magnetic, electrostatic, or both. If the barrier (shock overshoot) oscillates, then the protons would still be injected more efficiently than He ions, which does not seem to be supported by observations. Note, however, that the $\mathrm{He}^{++}$leakage from downstream has been finally obtained in simulations (Damiano Caprioli, this meeting). This finding may be an important step towards understanding the nature of backstreaming ions, including their composition.

Notwithstanding the remaining uncertainties, backstreaming protons of whatever origin are crucial for shock dissipation. They resonantly (and partially nonresonantly) drive the MHD waves whose saturation amplitude can be linked to the density of backstreaming protons. The latter was obtained in [8] as a function of the wave amplitude, and Mach number, thus making description self-consistent, at least in its proton part. In the first-order approximation, heavier elements are scattered by the proton-generated waves. The results of such calculations are shown in Fig 3 Although the proton spectra obtained in this model (not shown in the figure) compare very well with hybrid simulations available at the time [30], they were based on the backstreaming ions originating from the downstream fully thermalized Rankine-Hugoniot-compliant, hydrogen-dominated plasma.

The charge-sign dependence of injection also works against protons [8], but this time through the sign of the wave helicity, they drive upstream. The mechanism is that particle orbits that spiral along the perturbed magnetic field, have a preferred particle escape direction that depends on the charge sign $[8]$. So, $\bar{p}$ and $e^{-}$, for example, would have better chances for injection than $p$ and $e^{+}$, as they would preferentially escape

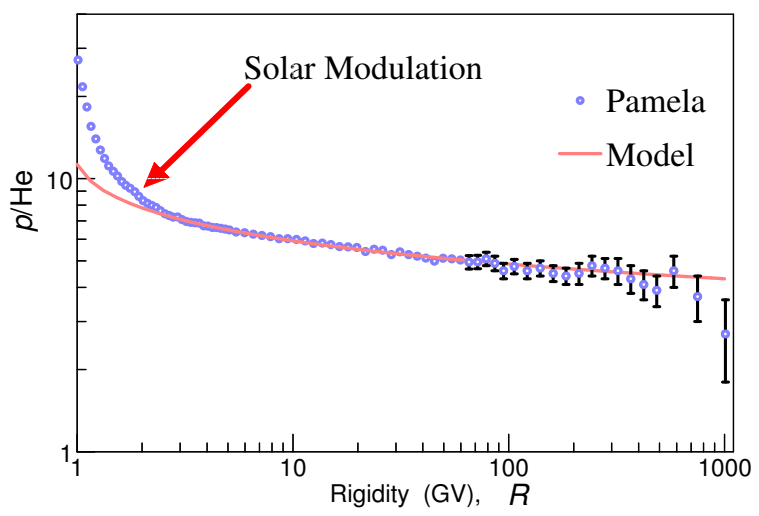

Figure 4: PAMELA points (circles) and theoretical fit from [6] (line). The twenty highest rigidity points are shown with the error-bars (stat.+syst.), where they seem to become significant. The difference between the data and theory at lower rigidities is due to the heliospheric modulation, not included in the model. This model would also fit the later AMS-02 data equally well, as they differ from those of Pamela only in normalization.

upstream through a wave generated by protons. However, both $\bar{p}$ and $e^{+}$need to be produced as secondaries in $p p$ collisions in the first place and remain available for further acceleration. I will argue in $\mathrm{Sec} 4$ that this combination of conditions is easier to fulfil for $e^{+}$than for $\bar{p}$. However, the mechanism will not be related to the wave-particle interaction.

Using the injection rates in Fig 3, the authors of Ref. [6] have suggested a solution to the problem of 0.1 difference in $\mathrm{He}$ and proton rigidity spectra that does not require any additional assumptions regarding SNR environments. A single spherical SNR shock, propagating in a homogeneous medium, must produce this difference. The focus of their explanation is on an initial (injection) phase of the DSA when the elemental similarity does not apply $\left(\mathcal{R}<\mathcal{R}_{0}\right)$. According to the injection theory [31, 8], collisionless shocks inject relatively more $\mathrm{He}^{2+}$ than protons when they are stronger. Naturally, they also produce harder spectra at that time, so the time integrated $\mathrm{He}^{2+}$ spectrum is harder as well. The result of convolution of the relative $p / \mathrm{He}$ injection rate with a typical SNR time history is illustrated in Fig 4.

\section{Modeling the $e^{+} / e^{-}$spectrum}

Model Requirements and Objectives. Similarly to the

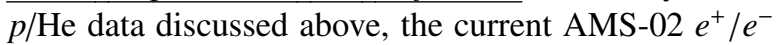
data, Fig, 1, have negligible statistical errors in an extended range between $0.5-100 \mathrm{GeV}$. The spectrum in this range is, however, considerably more complex, thus 
placing tighter constraints on the models. Therefore, a successful fit of the data in this range lends credence to the model predictions beyond it, including an interesting positron excess (and antiproton, for that matter) where the data are not so exact and even nonexistent at $E \gtrsim 500 \mathrm{GeV}$. Needless to say that an incipient bump around 200-300 GeV attracted much attention from the dark matter hunters.

Physics behind the Models. Most of the conventional scenarios for $e^{+} / e^{-}$excess invoke secondary positrons. They are produced by galactic CR protons in hadronic reactions. The collisions may occur in an ambient gas near an SNR accelerator, e.g. [32], elsewhere in the galaxy, e.g., [33, 34], or immediately in the SNR shock, thus being incorporated into the DSA, e.g. [35, 36]. Some of these scenarios face the unmatched antiprotons and other secondaries in the data, as discussed, e.g., in [37, 38, 39, 40]. Certain improvements along these lines have recently been achieved by using sophisticated Monte Carlo pp collision event generators, e.g. [41]. However, improved cross sections of $p p$ collisions do not shed more light on the physics of $e^{+} / e^{-}$anomaly, particularly the minimum at $8 \mathrm{GeV}$ and the U-shape form of the spectrum. This spectrum complexity hints at richer physics than a mere production of secondary $e^{+}$power-law spectra from the primary CR power-law.

Sources of positrons other than the secondaries from $p p$ collisions have also been suggested. Apart from the pulsars [42], the most intriguing are dark matter (DM) related scenarios [43, 44]. Regardless of the positron source considered, most of the models have enough "knobs" to fit the data. Some SNR based approaches, e.g., [45] directly use the AMS-02-measured pair of spectral indices to reproduce the U-shape spectrum in Fig 1 by adjusting the weights of the source and background contributions. The position of the spectral minimum also needs to be taken directly from the AMS02 data. Therefore, the physical nature of the minimum is not convincingly interpreted by these models, thus giving no credence to their predictions concerning the higher-energy positron excess. It follows that the model conclusions about a possible room for DM or lack thereof in the positron spectrum remain premature.

Of course, before embarking on the quest for DM, one should look for astrophysical interpretations of the $e^{+} / e^{-}$and $\bar{p} / p$ anomalies more carefully. The authors of [46] give an accurate and prosaic interpretation of namely the most precisely measured part of the positron fraction in the range $0.5-100 \mathrm{GeV}$. The explanation is based on the following two aspects of the DSA. First, the mechanism of positron injection is charge-sign

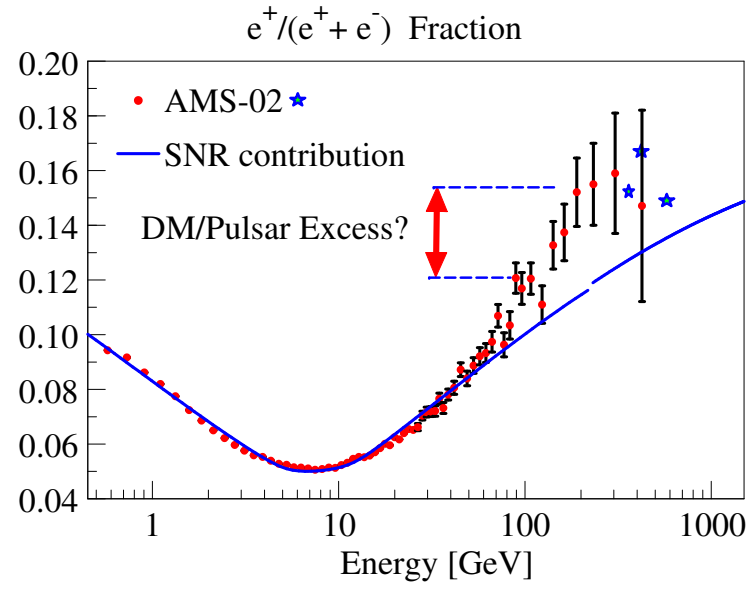

Figure 5: AMS-02 data [9], with the error bars added only where they are significant $(E>30 \mathrm{GeV})$. A few recent points (stars) from http://www.ams02.org/wp-content/uploads/2016/12/Final.pdf are included. Also shown is the positron fraction, obtained by solving the nonlinear acceleration problem. The predicted saturation level $\approx 0.17$.

asymmetric, which contrasts with our previous discussion of the $p-\mathrm{He}$ injection that primarily depends on the mass-to-charge ratio. They argue that the charge-sign selectivity of injection arises when the shock sweeps clumps of dense molecular gas (MC).

The second aspect of the mechanism proposed in [46] concerns a nonlinear shock modification by accelerated protons (NLDSA) which is known to make the momentum spectrum of low-energy particles steeper and that of the high-energy particles flatter than the canonical $p^{-4}$ spectrum produced by strong but unmodified shocks. Therefore, there is a point $p=p_{4}$ where the index is exactly four. Assuming most of galactic CR electrons to be accelerated in conventional shocks, thus having $p^{-4}$ source spectra, the ratio of the modified positron- to unmodified electron spectrum will show the required nonmonotonic behavior with a minimum at $p=p_{4}$. Note that, as in the case of $p / \mathrm{He}$ spectral anomaly, it is crucial to work with the positron/electron ratio, since the observed spectrum is automatically depropagated back to the source. In a customary $p^{4} f(p)$ normalization, the individual positron spectrum is the same as that of the $e^{+} / e^{-}$ratio which is, in turn, the same as the proton spectrum, provided that both species are accelerated to relativistic energies beyond their injection energies.

NLDSA. The nonlinear proton spectrum is well understood since it is solvable analytically [10, 47, 48]. Excellent agreement between the analytic and numerical solutions has been documented, e.g., in [11], Fig 1 . The 
analytic solution places the proton $p^{4} f(p)$ minimum at $\lesssim 10 \mathrm{GeV} / \mathrm{c}$ which is encouragingly consistent with the position of the observed $e^{+} / e^{-}$minimum. Moreover, it depends only weakly on the shock Mach number, $M$, proton's maximum momentum, $p_{\max }$, and their injection rate. However, $M \gtrsim 10$ and $p_{\max } / m_{p} c \gtrsim 500$ are the thresholds for a bifurcation into a strongly nonlinear (efficient acceleration) regime, shown in Fig 1, with its signature minimum around $5-10 \mathrm{GeV}$. Understanding of this transition requires an analytic form of the bifurcation diagram, obtained in [49, 48]. The latter cannot be inferred from most of the steady state numerical solutions, e.g. [12]. When the solution is not unique, it still converges to one particular branch and does not trace unstable manifolds, required to understand the bifurcation phenomenon (see below). The so-called semianalytic treatments, e.g. [50], do not provide the bifurcation diagram (commonly called the S-curve) either, as they do not solve the full problem in analytic form. The significance of the S-curve is that it encompasses three co-existing solutions in a certain range of the proton injection parameter $v_{1}<v_{\text {inj }}<v_{2}$, thus embodying hysteresis in this nonlinear system. It also provides two critical values for injection rates $v_{1,2}$ as functions of the Mach number $M$ and maximum momentum $p_{\max }$. One of the three solutions is unstable. The remaining two are, respectively, an inefficient solution, which corresponds to a test-particle solution at $v_{\text {inj }}<v_{1}$, and the efficient solution. For $v_{i n j}>v_{2}$ this solution is unique. Moreover, in the limit $M \gg 1$ and $p_{\max } \gg m_{p} c$ it can be obtained analytically and exactly [47], which is crucial in calculating the bifurcation diagram.

The solution co-existence domain between the critical injection rates $v_{1,2}\left(p_{\max }, M\right)$ is typically very narrow, $v_{2}-v_{1} \ll v_{1}$, which additionally prevents stationary numerical solutions from accessing the hysteretic shock behavior. By contrast, time-dependent numerical treatments should easily identify the transition from the inefficient branch at $v_{\text {inj }}<v_{1}$ to efficient one at $v_{\text {inj }}>v_{2}$. Indeed, when the system is evolved from a low $p_{\max } \lesssim m_{p} c$, at first, it sticks with the inefficient solution. With the (slowly) growing $p_{\max }$, the solution passes through the co-existence region, since both $v_{1}$ and $v_{2}>v_{1}$ decrease with $p_{\max }$. As soon as $v_{2}$ becomes $v_{2}<v_{\text {inj }}$, the only remaining system attractor (stationary solution under fixed $p_{\text {max }}$ ) is the efficient solution, to which the numerical time-dependent solution will quickly converge. This type of dynamics was likely seen in time-dependent kinetic simulations [51]. It occurred there in form of a series of sudden jumps to higher shock pre-compressions, manifesting the transitions from inefficient to efficient solutions when the former cease to exist $\left(v_{\mathrm{inj}}>v_{2}\right)$. This pattern of the shock evolution was predicted in [49, 48], including back transitions, though the authors of [51] attributed it to a likely numerical artifact. The incipient back transitions make the time-dependent evolution oscillatory in character, as seen in the simulations. The parameters of these limit-cycle oscillations (related to a Hopf bifurcation) depend on how other control parameters (primarily $v_{\text {inj }}$ ) respond to the changing shock compression during the forward or back transitions. This feedback loop is self-consistently included in the above simulations. Therefore, the limit-cycle type oscillations obtained in [51] are likely to be genuine and worth further study. It will help to understand the NLDSA dynamically, thus shedding new light on many shock-related astrophysical sources where a short-time variability is observed.

It should be noted, however, that a significant turbulent heating upstream is likely to straighten out the Scurve, thus making only one solution possible [48]. A similar effect of heating on the shock solutions occurs in some CR fluid models, e.g. [52]. The turbulent heating impacts the NLDSA in many ways but it is difficult to quantify, as the turbulence in the nonlinear shock precursor is still not well understood [53].

Interaction with MCs. Due to the sub-shock weakening in the NLDSA, MCs survive the ionizing radiation. Moreover, shock-accelerated CR protons illuminate the MC well before the subshock encounter. These CRs generate positrons and other secondaries in the MC interior by $p p$ collisions. They also charge the MC positively which creates a charge-sign asymmetry for the subsequent particle injection into the DSA via the following simple mechanism [46].

Because of a positive electrostatic potential in an MC, built by penetrating shock-accelerated protons, low-energy positively charged particles will be expelled from the MC, while negatively charged particles stay inside. Negatively-charged low-energy secondaries produced at the periphery of the MC by relatively lowenergy but abundant CR protons will be sucked into the MC. This phenomenon is particularly consistent with the $\bar{p} / p$ decline towards lower energies, Fig 2 However, for kinematic reasons of their generation and large mass ratio $m_{p} / m_{e}$, antiprotons will be absorbed by the MC much less efficiently than electrons. More importantly for the sequel, the same electric field expels the positively charged positrons from the MCs very efficiently. This is also consistent with an increase of the $e^{+} / e^{-}$ratio, towards lower energies Fig 1

The charging of MC by accelerated protons is a complex phenomenon [46] and merits a short discussion. 
While fully ionized plasmas are intolerant to external charges and immediately restore charge neutrality, sufficiently large and dense MCs respond differently. Due to a high rigidity of CRs, their density in the MC interior increases almost simultaneously with their density outside, when a CR-loaded shock approaches the MC. However, by contrast with a strongly ionized exterior, where the plasma resistivity is negligible, the electronion collisions inside the MC provide sufficient resistivity to neutralizing electric currents. Therefore, a strong macroscopic electric field will build up in response to the CR penetration, to neutralize the CR charge. This field expels the secondary positrons most efficiently as the lightest positively charged species - although it also shields the MC from low-energy CR protons.

The mechanism outlined above implies that negatively charged primaries and secondaries have much better chances to stay in an MC than positively charged particles. When the subshock eventually reaches the MC, the subshock engulfs it, e.g., [54, 55]. What was in the MC interior, is transferred downstream, largely unprocessed by the subshock. Therefore, the negatively charged particles mostly evade acceleration which explains why there is no $\bar{p} / p$ excess similar to that of $e^{+} / e^{-}$at high energies, cf. Figs 5 and 2 . Also, antiprotons are strongly depleted towards lower energies, since they are likely to be trapped by the MC electrostatic potential, as discussed above. Antiprotons born in $p p$ collisions with higher energies (typically a few $\mathrm{GeV}$ ) can be retained in the MC only if their are deep inside. Otherwise, they are accelerated just the way the protons are, which explains the flat $\bar{p} / p$ ratio at higher energies.

\section{Conclusions and Outlook}

The common belief that the $\mathrm{CR}$ rigidity spectrum is a power-law with only a few distinct structures (e.g., knee and ankle), the same for all primary elements, is being rapidly abandoned. New data have challenged the idea of acceleration scalability on the entire rigidity range of a given accelerator, such as an SNR.

Multiple sources (SNRs) with adjustable spectra or sources with poorly known spectra (DM, pulsars, superbubbles with uncertain environments) were almost universally proposed to accommodate the new spectral features. By contrast, the gist of this paper was that a single-SNR CR acceleration allows for much more certain predictions about rigidity spectra of different elements. Moreover, some SNRs are accessible to direct observations across a broad emission spectrum. The CR production in such sources, including the $\mathrm{CR}$ interaction with adjacent MCs [56], is therefore reasonably well understood which provides further insights into the nature of CR anomalies discussed in this paper. It was demonstrated that it is possible to fit a high-fidelity part of the data, provided that the following elements of the DSA physics are addressed

- self-consistent particle injection, depending on mass to charge ratio and shock Mach number

- clumps of a dense gas present in SNR shock environments

- backreaction of accelerated protons on the shock and all particle injection and acceleration

It is clear that the proposed single-source mechanisms equally apply to an ensemble of similar SNRs without introducing additional free parameters. A possible exception is an upturn in $p$-He rigidity spectra near 300 $\mathrm{GV}$, not discussed in this paper. Its most obvious explanation is that a steeper, low-energy part of the spectrum is produced by a nearby SNR. At rigidities $>300 \mathrm{GV}$, it "sinks" into a slightly harder spectrum, which is either created by a stronger (younger) but more remote object, or else represents galactic background. The relative normalization of the two contributions needs then to be adjusted to place the kink in the right place in the spectrum. The upturn, though, might also be explained by a CR self-confinement effect around the source via CR-emitted Alfven waves [57]. It is not clear, however, if the latter scenario can be elaborated with a lesser number of free parameters than the two-source model. If the two-source scenario is at work, the following conclusion, relevant to the present study, can be drawn from the $300 \mathrm{GV}$ upturn. Because the $p / \mathrm{He}$ spectrum does not show any change in its slope at this rigidity, the mechanism of $p$ and He injection in both sources is likely to be universal, as argued in this paper.

Further insights into the physics of injection give much improved numerical simulations. However, as they are becoming more realistic, their physical understanding is often a challenge. The present day simulations can be considered as numerical experiments, not much easier to interpret than real observations. Understanding their limitations by benchmarking between different software suites and comparison with simplified physical models is a promising avenue to understanding rapidly improving observations.

To conclude, I briefly dwell on the prospects for resolving the remaining mismatch with the positron fraction in the 200-400 GeV range, Fig.5. Assuming that the data, notwithstanding error bars in this range, are 
statistically significant, one may suggest a few explanations of this deviation. The most exciting one is that the limited-energy-range excess over the SNR positron production manifests a DM annihilation or decay. Ironically, the model described in this paper that was incited to account for the data astrophysically facilitates the DM scenario by providing an SNR "background". It significantly eases the requirements for the positron production by DM particles. Not going deeper into DM scenarios, several conventional ways to explain the deviation, may also be suggested [46]. Further elaborations on them may or may not close the remaining gap between the SNR positron production and the AMS-02 data. For now, the possibility of DM or pulsar contribution to the positron excess cannot be ruled out, so the prospects for the future of the CR research are exciting.

\section{Acknoledgements}

I'm grateful to the organizers for inviting me to speak at this meeting. I also thank Roger Blandford, Igor Moskalenko, and Paul Simeon for useful discussions we have had afterward in Palo Alto, California.

This work was supported by the NASA Astrophysics Theory Program under Grant No. NNX14AH36G.

\section{References}

[1] R. Blandford, P. Simeon, Y. Yuan, Cosmic Ray Origins: An Introduction, Nuclear Physics B Proceedings Supplements 256 (2014) 9-22. arXiv:1409.2589 doi:10.1016/j. nuclphysbps.2014.10.002

[2] A. E. Vladimirov, G. Jóhannesson, I. V. Moskalenko, T. A. Porter, Testing the Origin of High-energy Cosmic Rays, Astrophys. J. 752 (2012) 68. arXiv:1108.1023 doi:10.1088/ 0004-637X/752/1/68

[3] M. Aguilar, L. Ali Cavasonza, B. Alpat, et al., Antiproton Flux, Antiproton-to-Proton Flux Ratio, and Properties of Elementary Particle Fluxes in Primary Cosmic Rays Measured with the Alpha Magnetic Spectrometer on the International Space Station, Physical Review Letters 117 (9) (2016) 091103. doi: 10.1103/PhysRevLett.117.091103

[4] O. Adriani, G. C. Barbarino, G. A. Bazilevskaya, et al., PAMELA Measurements of Cosmic-Ray Proton and Helium Spectra, Science 332 (2011) 69-. arXiv:1103.4055 doi: 10.1126/science.1199172

[5] Y. S. Yoon, H. S. Ahn, P. S. Allison, et al., Cosmic-ray Proton and Helium Spectra from the First CREAM Flight, Astrophys. J. 728 (2011) 122. arXiv:1102.2575 doi:10.1088/ 0004-637X/728/2/122

[6] M. A. Malkov, P. H. Diamond, R. Z. Sagdeev, Proton-Helium Spectral Anomaly as a Signature of Cosmic Ray Accelerator, Physical Review Letters 108 (8) (2012) 081104. arXiv:1110. 5335 doi:10.1103/PhysRevLett.108.081104

[7] Y. Ohira, N. Kawanaka, K. Ioka, Cosmic-ray hardenings in light of AMS-02 data, Phys. Rev. D93 (8) (2016) 083001. arXiv: 1506.01196 doi:10.1103/PhysRevD.93.083001
[8] M. A. Malkov, Ion leakage from quasiparallel collisionless shocks: Implications for injection and shock dissipation, Phys. Rev. E 58 (1998) 4911-4928.

[9] L. Accardo, M. Aguilar, D. Aisa, et al., High Statistics Measurement of the Positron Fraction in Primary Cosmic Rays of 0.5$500 \mathrm{GeV}$ with the Alpha Magnetic Spectrometer on the International Space Station, Physical Review Letters 113 (12) (2014) 121101. doi:10.1103/PhysRevLett.113.121101

[10] M. A. Malkov, Analytic Solution for Nonlinear Shock Acceleration in the Bohm Limit, Astrophys. J. 485 (1997) 638-654. doi: $10.1086 / 304471$

[11] I. V. Moskalenko, T. A. Porter, M. A. Malkov, P. H. Diamond, Hadronic Gamma Rays from Supernova Remnants, ArXiv eprints 705. arXiv:0705.3854

[12] D. C. Ellison, E. G. Berezhko, M. G. Baring, Nonlinear Shock Acceleration and Photon Emission in Supernova Remnants, Astrophys. J. 540 (2000) 292-307. doi :10.1086/309324

[13] O. Adriani, G. C. Barbarino, G. A. Bazilevskaya, et al., An anomalous positron abundance in cosmic rays with energies 1.5-100GeV, Nature458 (2009) 607-609. arXiv:0810.4995. doi:10.1038/nature07942

[14] M. Aguilar, G. Alberti, B. Alpat, et al., First Result from the Alpha Magnetic Spectrometer on the International Space Station: Precision Measurement of the Positron Fraction in Primary Cosmic Rays of 0.5-350 GeV, Physical Review Letters 110 (14) (2013) 141102. doi:10.1103/PhysRevLett.110.141102

[15] N. Arkani-Hamed, D. P. Finkbeiner, T. R. Slatyer, N. Weiner, A theory of dark matter, Phys. Rev. D79 (1) (2009) 015014. arXiv:0810.0713 doi:10.1103/PhysRevD.79.015014

[16] V. Berezinsky, V. Dokuchaev, Y. Eroshenko, SUSY dark matter annihilation in the Galactic halo, Journal of Physics Conference Series 607 (1) (2015) 012015. arXiv: 1506.03955 doi: 10. 1088/1742-6596/607/1/012015

[17] P. D. Serpico, Possible physics scenarios behind cosmic-ray "anomalies", ArXiv 1509.04233 arXiv: 1509.04233

[18] M. Aguilar, D. Aisa, B. Alpat, et al., Precision Measurement of the Helium Flux in Primary Cosmic Rays of Rigidities 1.9 GV to 3 TV with the Alpha Magnetic Spectrometer on the International Space Station, Physical Review Letters 115 (21) (2015) 211101. doi:10.1103/PhysRevLett.115.211101

[19] N. Tomassetti, Origin of the Proton-to-helium Ratio Anomaly in Cosmic Rays, Astrophys. J. Lett. 815 (2015) L1. arXiv: 1511.04460 doi:10.1088/2041-8205/815/1/L1

[20] M. Bertaina, W. Apel, J. Arteaga-Velazquez, et al., The cosmic ray spectrum and composition measured by

kascade-grande between 1016 ev and 1018 ev Nuclear Physics B - Proceedings Supplements 256 (2014) 149 - 160. doi:http://dx.doi.org/10.1016/j.nuclphysbps . 2014.10.018

URL http://www.sciencedirect.com/science/ article/pii/S0920563214002126

[21] P. L. Biermann, J. K. Becker, J. Dreyer, et al., The Origin of Cosmic Rays: Explosions of Massive Stars with Magnetic Winds and Their Supernova Mechanism, Astrophys. J. 725 (2010) 184187. arXiv:1009.5592 doi:10.1088/0004-637X/725/1/ 184

[22] D. C. Ellison, L. O. Drury, J.-P. Meyer, Galactic Cosmic Rays from Supernova Remnants. II. Shock Acceleration of Gas and Dust, Astrophys. J. 487 (1997) 197-217. arXiv:astro-ph/ 9704293 doi:10.1086/304580

[23] K. B. Quest, Theory and simulation of collisionless parallel shocks, J. Geophys. Res. 93 (1988) 9649-9680.

[24] E. N. Parker, A quasi-linear model of plasma shock structure in a longitudinal magnetic field, Journal of Nuclear Energy 2 (1961) 146-153. 
[25] D. Burgess, E. Möbius, M. Scholer, Ion Acceleration at the Earth's Bow Shock, Space Sci. Rev. (2012) 5-47doi :10.1007/ s11214-012-9901-5

[26] D. Caprioli, A.-R. Pop, A. Spitkovsky, Simulations and Theory of Ion Injection at Non-relativistic Collisionless Shocks, Astrophys. J. Lett. 798 (2015) L28. arXiv:1409.8291. doi: 10.1088/2041-8205/798/2/L28

[27] G. M. Zaslavsky, Physics of chaos in Hamiltonian systems, 2007.

[28] T. V. Liseykina, G. I. Dudnikova, V. A. Vshivkov, M. A. Malkov, Ion-acoustic shocks with reflected ions: modelling and particle-in-cell simulations Journal of Plasma Physics 81 . doi:10.1017/S002237781500077X

URL http://journals.cambridge.org/article_ S002237781500077X

[29] M. A. Malkov, R. Z. Sagdeev, G. I. Dudnikova, et al., Ion-acoustic shocks with self-regulated ion reflection and acceleration Physics of Plasmas 23 (4). doi:http://dx.doi.org/10.1063/1.4945649

URL http://scitation.aip.org/content/aip/ journal/pop/23/4/10.1063/1.4945649

[30] L. Bennett, D. C. Ellison, Investigation of intrinsic variability in one-dimensional parallel shocks using steady state hybrid simulations, J. Geophys. Res. 100 (1995) 3439-3448. doi: 10.1029/94JA02579

[31] M. A. Malkov, H. J. Völk, Theory of ion injection at shocks., Astronomy and Astrophys.300 (1995) 605-626.

[32] Y. Fujita, K. Kohri, R. Yamazaki, K. Ioka, Is the PAMELA anomaly caused by supernova explosions near the Earth?, Phys. Rev. D80 (6) (2009) 063003. arXiv:0903.5298, doi:10. 1103/PhysRevD.80.063003

[33] K. Blum, B. Katz, E. Waxman, AMS-02 Results Support the Secondary Origin of Cosmic Ray Positrons, Physical Review Letters 111 (21) (2013) 211101. arXiv:1305.1324 doi:10. 1103/PhysRevLett.111.211101

[34] R. Cowsik, B. Burch, T. Madziwa-Nussinov, The Origin of the Spectral Intensities of Cosmic-Ray Positrons, Astrophys. J. 786 (2014) 124. arXiv:1305.1242 doi:10.1088/0004-637X/ $786 / 2 / 124$

[35] P. Blasi, Origin of the Positron Excess in Cosmic Rays, Physical Review Letters 103 (5) (2009) 051104. arXiv : 0903.2794 doi:10.1103/PhysRevLett.103.051104

[36] P. Mertsch, S. Sarkar, Testing Astrophysical Models for the PAMELA Positron Excess with Cosmic Ray Nuclei, Physical Review Letters 103 (8) (2009) 081104. arXiv:0905.3152 doi:10.1103/PhysRevLett.103.081104

[37] I. V. Moskalenko, A. W. Strong, J. F. Ormes, M. S. Potgieter, Secondary Antiprotons and Propagation of Cosmic Rays in the Galaxy and Heliosphere, Astrophys. J. 565 (2002) 280-296. arXiv:astro-ph/0106567 doi:10.1086/324402

[38] M. Kachelrieß, S. Ostapchenko, R. Tomàs, Antimatter Production in Supernova Remnants, Astrophys. J. 733 (2011) 119 arXiv:1103.5765 doi:10.1088/0004-637X/733/2/119

[39] I. Cholis, D. Hooper, Constraining the origin of the rising cosmic ray positron fraction with the boron-to-carbon ratio, Phys. Rev. D89 (4) (2014) 043013. arXiv:1312.2952 doi:10. 1103/PhysRevD.89.043013

[40] Q. Yuan, S.-J. Lin, K. Fang, X.-J. Bi, Propagation of cosmic rays in the AMS-02 era, ArXiv e-prints arXiv:1701.06149

[41] K. Kohri, K. Ioka, Y. Fujita, R. Yamazaki, Can we explain AMS-02 antiproton and positron excesses simultaneously by nearby supernovae without pulsars or dark matter?, Progress of Theoretical and Experimental Physics 2016 (2) (2016) 021E01. arXiv:1505.01236 doi:10.1093/ptep/ptv193

[42] S. Profumo, Dissecting cosmic-ray electron-positron data with
Occam's razor: the role of known pulsars, Central European Journal of Physics 10 (2012) 1-31. arXiv:0812.4457 doi: 10.2478/s11534-011-0099-z

[43] L. Bergström, T. Bringmann, I. Cholis, D. Hooper, C. Weniger, New Limits on Dark Matter Annihilation from Alpha Magnetic Spectrometer Cosmic Ray Positron Data, Physical Review Letters 111 (17) (2013) 171101. arXiv:1306.3983 doi:10.1103/PhysRevLett.111.171101

[44] I. Cholis, D. Hooper, Dark matter and pulsar origins of the rising cosmic ray positron fraction in light of new data from the AMS, Phys. Rev. D88 (2) (2013) 023013. arXiv:1304.1840 doi: 10.1103/PhysRevD.88.023013

[45] A. D. Erlykin, A. W. Wolfendale, Cosmic ray positrons from a local, middle-aged supernova remnant, Astroparticle Physics 49 (2013) 23-27. arXiv:1308.4878 doi:10.1016/j. astropartphys.2013.08.001

[46] M. Malkov, P. Diamond, R. Sagdeev, Positive charge prevalence in cosmic rays: Room for dark matter in the positron spectrum, Physical Review D 94 (6) (2016) 063006.

[47] M. A. Malkov, Asymptotic Particle Spectra and Plasma Flows at Strong Shocks, Astrophys. J. Lett. 511 (1999) L53-L56. doi: 10.1086/311825

[48] M. A. Malkov, L. O. Drury, Nonlinear theory of diffusive acceleration of particles by shock waves, Reports on Progress in Physics 64 (2001) 429-481.

[49] M. A. Malkov, Bifurcation, Efficiency, and the Role of Injection in Shock Acceleration with the Bohm Diffusion, Astrophys. $J$. 491 (1997) 584-595. doi:10.1086/304990

[50] P. Blasi, A semi-analytical approach to non-linear shock acceleration, Astroparticle Physics 16 (2002) 429-439. arXiv: arXiv:astro-ph/0104064

[51] H. Kang, T. W. Jones, Acceleration of Cosmic Rays at Large Scale Cosmic Shocks in the Universe, Journal of Korean Astronomical Society 35 (2002) 159-174. arXiv:astro-ph/ 0211360 doi:10.5303/JKAS.2002.35.4.159

[52] P. Mostafavi, G. P. Zank, G. M. Webb, Pickup ion mediated plasmas: Shock wave structure, in: American Institute of Physics Conference Series, Vol. 1720 of American Institute of Physics Conference Series, 2016, p. 090003. doi: 10.1063/1.4943855

[53] M. A. Malkov, P. H. Diamond, R. Z. Sagdeev, On the structure and scale of cosmic ray modified shocks, Plasma Physics and Controlled Fusion 52 (12) (2010) 124006-+. arXiv: 1007.3042 doi:10.1088/0741-3335/52/12/124006

[54] T. Inoue, R. Yamazaki, S.-i. Inutsuka, Y. Fukui, Toward Understanding the Cosmic-Ray Acceleration at Young Supernova Remnants Interacting with Interstellar Clouds: Possible Applications to RXJ 1713.7-3946, Astrophys. J. 744 (2012) 71. arXiv:1106.3080 doi:10.1088/0004-637X/744/1/71

[55] B. Draine, Physics of the Interstellar and Intergalactic Medium Princeton Series in Astrophysics, Princeton University Press, 2010.

URL FycJvKHyiwsC

[56] M. A. Malkov, P. H. Diamond, R. Z. Sagdeev, Mechanism for spectral break in cosmic ray proton spectrum of supernova remnant W44, Nature Communications 2/1195. arXiv: 1004.4714 doi:10.1038/ncomms1195

[57] M. A. Malkov, P. H. Diamond, R. Z. Sagdeev, F. A. Aharonian, I. V. Moskalenko, Analytic Solution for Self-regulated Collective Escape of Cosmic Rays from Their Acceleration Sites, Astrophys. J. 768 (2013) 73. arXiv:1207.4728 doi: 10.1088/0004-637X/768/1/73 